\title{
Special Issue on Spatial Multivariate Methods
}

\author{
Eric Grunsky
}

Received: 13 April 2012 / Accepted: 17 April 2012 / Published online: 27 April 2012

(C) International Association for Mathematical Geosciences 2012

This special issue is dedicated to spatial multivariate methods and addresses the emergence of the latest practical and theoretical aspects on this topic. The papers herein present a wide spectrum of recent advances in methods and applications in diverse areas. It grew out of an invited paper session of the International Statistical Institute meeting that was held in Durban, South Africa, in 2009. This special issue, comprised of seven papers, a teaching aid and short note, aims to broaden interest and offer insights into current trends and future directions of spatial multivariate methods.

The issue begins with Bailey and Krzanowski's overview of the existing approaches for the analysis of geostatistical multivariate data. These approaches are divided into classes of factor models or spatial random field models; and their differences are discussed. Their paper introduces and discusses the minimum/maximum autocorrelation factors (MAF), which is a common theme throughout this special issue. The next paper by Christensen and Sain considers an alternate view of multimodel ensembles for use with the North American Regional Climate Change Assessment Program. Numerical models of atmosphere-ocean circulation are widely used to understand past climate and to project future climate change. The authors propose a spatially-correlated latent variable model in order to facilitate the exploration of similarities between regional climate models and what factors best predict observed locations of model convergence.

In their paper, Mueller and Ferreira contrast the MAF method with the Gauss iterations (U-WEDGE) method and present a comparison with a full cosimulation of the attributes. Du and Ma propose an efficient approach in constructing variogram matrix functions in order to measure the dependence of a vector random field with secondorder increments. This paper further demonstrates that various dependence structures

E. Grunsky $(\bowtie)$

Geological Survey of Canada, Ottawa, Ontario, Canada

e-mail: egrunsky@nrcan.gc.ca 
among components can be derived through appropriate mixture procedures. Boucher and Dimitrakopoulos' paper presents the application of block-support simulation of a multi-element mineral deposit based on MAF so as to facilitate the computationally efficient joint simulation of large, multivariate mineral deposits. An algorithm is developed and utilized successfully at an iron ore deposit to simulate five crosscorrelated elements that are critical in defining the quality of iron ore being mined. In a short note, Oman and Vakulenko-Lagun show that an iterative algorithm for estimating sill matrices for multivariate geostatistical data is a minimization problem that is guaranteed to converge and thereby allowing for a solution in a linear model of co-regionalization. Finally, in a teaching aid, Rondon provides a detailed review of the MAF factorization and its applications and guides practitioners on the method's use through examples.

The papers included here demonstrate the expertise in the field of spatial multivariate methods. It is the hope that this issue will provide a thorough overview of this topic and stimulate further interest in the subject. 